\title{
Elevated Type 1 Metabotropic Glutamate Receptor Availability in a Mouse Model of Huntington's Disease: a Longitudinal PET Study
}

\author{
Daniele Bertoglio $^{1}$ (D) Jeroen Verhaeghe ${ }^{1} \cdot$ Špela Korat $^{1,2} \cdot$ Alan Miranda $^{1} \cdot$ Klaudia Cybulska $^{1,2} \cdot$ Leonie Wyffels $^{1,2}$. \\ Sigrid Stroobants ${ }^{1,2} \cdot$ Ladislav Mrzljak $^{3}$ - Celia Dominguez ${ }^{3}$. Mette Skinbjerg ${ }^{3}$ - Longbin Liu $^{3}$. \\ Ignacio Munoz-Sanjuan ${ }^{3} \cdot$ Steven Staelens ${ }^{1}$
}

Received: 4 October 2019 / Accepted: 22 December 2019 / Published online: 8 January 2020

(C) The Author(s) 2020

\begin{abstract}
Impairment of group I metabotropic glutamate receptors (mGluRs) results in altered glutamate signalling, which is associated with several neurological disorders including Huntington's Disease (HD), an autosomal neurodegenerative disease. In this study, we assessed in vivo pathological changes in mGluR1 availability in the Q175DN mouse model of HD using longitudinal positron emission tomography (PET) imaging with the radioligand $\left[{ }^{11} \mathrm{C}\right] \mathrm{ITDM}$. Ninety-minute dynamic PET imaging scans were performed in 22 heterozygous (HET) Q175DN mice and 22 wild-type (WT) littermates longitudinally at 6, 12, and 16 months of age. Analyses of regional volume of distribution with an image-derived input function $\left(V_{\mathrm{T}}\right.$ (IDIF) $)$ and voxel-wise parametric $V_{\mathrm{T}}$ (IDIF) maps were performed to assess differences between genotypes. Post-mortem evaluation at 16 months was done to support in vivo findings. $\left[{ }^{11} \mathrm{C}\right] \mathrm{ITDM} V_{\mathrm{T}}$ (IDIF) quantification revealed higher mGluR1 availability in the brain of HET mice compared to WT littermates (e.g. cerebellum: $+15.0 \%,+17.9 \%$, and $+17.6 \%$ at 6,12 , and 16 months, respectively; $p<0.001$ ). In addition, an age-related decline in $\left[{ }^{11} \mathrm{C}\right]$ ITDM binding independent of genotype was observed between 6 and 12 months. Voxel-wise analysis of parametric maps and post-mortem quantifications confirmed the elevated mGluR1 availability in HET mice compared to WT littermates. In conclusion, in vivo measurement of mGluR1 availability using longitudinal $\left[{ }^{11} \mathrm{C}\right]$ ITDM PET imaging demonstrated higher $\left[{ }^{11} \mathrm{C}\right] \mathrm{ITDM}$ binding in extra-striatal brain regions during the course of disease in the Q175DN mouse model.
\end{abstract}

Keywords $\left[{ }^{11} \mathrm{C}\right] \mathrm{ITDM} \cdot$ Glutamate $\cdot \mathrm{mGluR} 1 \cdot$ Brain imaging $\cdot$ Receptor

\section{Introduction}

Glutamate is the primary excitatory neurotransmitter in the central nervous system and exerts its action through both ionotropic and metabotropic receptors. Group I metabotropic glutamate receptors (mGluRs) are post-synaptic G proteincoupled receptors and include mGluR type 1 (mGluR1) and type 5 (mGluR5) [1]. These receptors mainly promote

Electronic supplementary material The online version of this article (https://doi.org/10.1007/s12035-019-01866-5) contains supplementary material, which is available to authorized users.

Steven Staelens

steven.staelens@uantwerpen.be

1 Molecular Imaging Center Antwerp (MICA), University of Antwerp, Wilrijk, Belgium

2 Department of Nuclear Medicine, Antwerp University Hospital, Edegem, Belgium

3 CHDI Management/CHDI Foundation, Los Angeles, CA, USA intracellular $\mathrm{Ca}^{2+}$ release and protein kinase $\mathrm{C}$ activation [2]. Impairment in their functionality can cause altered glutamate signalling and consequently excitotoxicity, a reason why both mGluR1 and mGluR5 have been associated with several neurological disorders, including Huntington's Disease (HD) [3, 4].

$\mathrm{HD}$ is an inherited autosomal dominant neurodegenerative disorder caused by an expanded polyglutamine (CAG) repeat in the exon 1 of the HTT gene [5]. This mutated gene translates to mutant huntingtin (mHTT), which is the causative agent of the disease. As a consequence of mHTT accumulation, neuronal dysfunction and death occurs, leading to progressive motor, psychiatric, and cognitive impairments in individuals with $\mathrm{HD}[6,7]$.

Of note, mGluR1 and mGluR5 display distinct cerebral expression patterns: mGluR5 is highly expressed in striatum, hippocampus, and cortex, whereas mGluR1 has a primarily thalamic and cerebellar distribution, with low levels in the other brain regions $[8,9]$.

Thus, likely due to the striatal and cortical distribution, previous studies mainly focused on mGluR5 and described 
altered receptor density in both HD mice [10] and human postmortem tissue [11], although an understanding of the underlying mechanism is still a topic of debate. Knockout and pharmacological antagonism of mGluR5 have been shown to reduce formation of mHTT aggregates [12,13], whilst mGluR5 positive allosteric modulation improved synaptic plasticity $[14,15]$. Additionally, we recently characterized the longitudinal changes of mGluR5 density occurring during disease progression in the Q175 mouse model of HD [16] by means of positron emission tomography (PET) and found that mGluR5 levels were decreased in HD mice [17].

On the contrary, mGluR1 has received limited attention, and little is known on possible changes in mGluR1 availability during the progression of HD. However, the extra-striatal distribution of mGluR1 is exactly in regions implicated in movement disorders, such as the cerebellum and thalamus, and it is thus of high relevance, among others, to HD [3]. The aim of this study was to investigate whether changes in mGluR1 availability occur during disease progression in the same mouse model of HD we used to quantify mGluR5 [17] in order to provide a first evidence before clinical investigation. We performed longitudinal non-invasive PET imaging of mGluR1 at 6,12 , and 16 months of age using the selective radioligand $\left[{ }^{11} \mathrm{C}\right] \mathrm{ITDM}$ (N-[4-[6-(isopropylamino)pyrimidin-4-yl]-1,3-thiazol-2-yl]-N-methyl-4-[ $\left.{ }^{11} \mathrm{C}\right]-$ methylbenzamide) [18], for which we recently validated the pharmacokinetic methodology to perform accurate quantification of mGluR1 availability in the mouse brain [19]. By using the same Q175 mouse model, these findings will offer new insights into the characterization of both mGluR1 and mGluR5 during HD progression.

\section{Materials and Methods}

\section{Animals}

Thirty-seven 6 months old male heterozygous (HET) Q175DN mice (C57BL/6J background) and 37 age-matched wild-type (WT) littermates were obtained from the Jackson Laboratories (Bar Harbour, Maine, USA). Since C57BL/6J mice present sporadic congenital portosystemic shunt [20], animals were screened before inclusion in the study to avoid this variable as confounding factor. This mouse model of HD exhibits motor, cognitive, molecular, and electrophysiological abnormalities, including in vivo decrease in several striatal markers and HD hallmarks similarly to patients with HD $[16,17,21-24]$. Only HET mice were included in the study to better resemble the clinical condition as homozygousity is rare in patients with HD.

Twenty-two mice per genotype were allocated to the longitudinal study, while the remaining ones ( $n=15 /$ genotype) were used for radiometabolite measurements. Animals were single-housed in individually ventilated cages with temperature- and humidity-controlled environment on a $12 \mathrm{~h}$ light/dark cycle with access to food and water ad libitum. Animals were given at least 1 week to habituate to the facility before the start of the procedures.

\section{Radiotracer Synthesis}

$\left[{ }^{11} \mathrm{C}\right]$ ITDM synthesis was performed on an automated synthesis module (Carbosynthon I, Comecer, The Netherlands) optimizing the reported procedure [18] to our system as recently described [19]. Average molar activity was $90.1 \pm 27.6 \mathrm{GBq} /$ $\mu$ mol with radiochemical purity greater than $99 \%$. $\left[{ }^{3} \mathrm{H}\right] \mathrm{ITDM}$ was synthetized by Pharmaron, UK, with a molar radioactivity of $2.66 \mathrm{GBq} / \mu \mathrm{mol}$ and purity above $99.9 \%$.

\section{$\left[{ }^{11}\right.$ C]ITDM Dynamic PET Imaging}

\section{Image Acquisition}

Dynamic microPET/computed tomography (CT) imaging was performed on two Siemens Inveon PET/CT scanners (Siemens Preclinical Solution, Knoxville, USA). Following isoflurane anaesthesia (induction 5\%, maintenance 1.5-2\% supplemented in oxygen) (Forene, Belgium), animals were catheterized in the tail vein for intravenous (i.v.) bolus injection of the tracer and were placed on the scanner bed. The whole body was in the PET scanner's field of view to allow the extraction of the image-derived input function (IDIF) from the lumen of the left ventricle of the heart as previously described and validated $[19,25]$. A monitoring acquisition module (Minerve, France) was used to constantly monitor the respiration of the animal during the entire preparation and scanning period. The core body temperature of the animals was maintained stable at $37 \pm 1^{\circ} \mathrm{C}$ using a heating pad during the entire preparation phase and a feedback-controlled warm air flow (Minerve, France) during the scanning period.

$\left[{ }^{11} \mathrm{C}\right]$ ITDM image acquisition was performed as previously validated [19]. At the onset of the 90 min dynamic microPET scan, animals were injected with a bolus of $\left[{ }^{11} \mathrm{C}\right]$ ITDM over a $12 \mathrm{~s}$ interval $(1 \mathrm{ml} / \mathrm{min})$ using an automated pump (Pump 11 Elite, Harvard Apparatus, USA). Activity was injected in a trace dose, keeping the cold mass similar across time points, and below a maximum limit $(2.0 \mu \mathrm{g} / \mathrm{kg})$. PET scans were acquired in list-mode format and followed by a $10 \mathrm{~min} 80 \mathrm{kV} /$ $500 \mu \mathrm{A} \mathrm{CT}$ scan for attenuation correction and co-registration.

Animals $(n=44)$ were imaged longitudinally at 6,12 , and 16 months of age. A total of $2 \mathrm{WT}$ and 4 HET Q175DN mice died during longitudinal imaging (in part due to recovery related to anaesthesia). Details regarding body weight, molar radioactivity, injected radioactivity and mass, and number of animals for each time point are provided in Supplementary Table 1. 


\section{Metabolite Analysis}

In order to assess possible genotypic difference in peripheral metabolism, a population-based metabolite analysis was performed in a cohort of WT $(n=15)$ and HET $(n=15)$ mice at 0 , 5,15 , and $30 \mathrm{~min}$ post injection (p.i.). The procedure was as previously described [19]. Briefly, mice were injected with the radioligand via the lateral tail vein, and blood was collected via cardiac puncture. Next, samples were centrifuged at $2377 \times$ rcf for $5 \mathrm{~min}$, and both plasma and residual fractions were counted in a gamma counter (Wizard ${ }^{2}$, PerkinElmer). Following addition of equal amounts of ice-cold acetonitrile to the plasma samples and centrifugation at $2377 \times \mathrm{rcf}$ for 5 min, supernatant was separated from the precipitate, and both fractions were counted in the gamma counter to calculate the plasma extraction efficiency (WT: $96.4 \pm 2.3 \%$; HET: $97.3 \pm 0.8 \%$ ). Finally, $100 \mu \mathrm{l}$ of supernatant were loaded onto a preconditioned reverse-phase (RP)-HPLC system (Kinetex, $150 \times 4.6 \mathrm{~mm}, 5 \mu \mathrm{m}$ HPLC column + Phenomenex security guard precolumn), fractions were collected, and radioactivity was measured in the gamma counter to determine the unchanged fraction. Blood spiked in vitro with $37 \mathrm{kBq}$ of radiotracer indicated no degradation occurred during the workup (unchanged radioligand $=99.3 \pm 0.4 \%$ ).

\section{Image Processing and Data Analysis}

Acquired 90 min PET data were histogrammed and reconstructed into 39 frames of increasing length $(12 \times 10 \mathrm{~s}, 3 \times$ $20 \mathrm{~s}, 3 \times 30 \mathrm{~s}, 3 \times 60 \mathrm{~s}, 3 \times 150 \mathrm{~s}$, and $15 \times 300 \mathrm{~s}$ ). Images were reconstructed using a list-mode iterative reconstruction with proprietary spatially variant resolution modelling with 8 iterations and 16 subsets of the 3D ordered subset expectation maximization (OSEM 3D) algorithm. Normalization, dead time, and CT-based attenuation corrections were applied. PET image frames were reconstructed on a $128 \times 128 \times 159$ grid with $0.776 \times 0.776 \times 0.776 \mathrm{~mm}^{3}$ voxels. Processing and analysis of the PET images was performed using PMOD 3.6 software (Pmod Technologies, Zurich, Switzerland).

Spatial normalization of the PET images was achieved by normalization of the images to a $\left[{ }^{11} \mathrm{C}\right]$ ITDM PET template as previously described [19, 24]. Time activity curves (TACs) were extracted from the images using a volume of interest brain atlas adapted from the Waxholm one [26]. mGluR1 is primarily expressed in cerebellum and thalamus. Nonetheless, it can be measured at lower levels also in other brain regions, so we considered striatum, motor cortex, and hippocampus given their relevance to HD. Finally, pons was included in order to assess the application of reference region-based analysis.

Kinetic modelling was performed by fitting the TACs using a two-tissue compartment model (2TCM) with a blood volume fraction $\left(V_{\mathrm{B}}\right)$ fixed at 3.6\% [27] and using the Logan model [28] with the start of the linear phase $\left(t^{*}\right)$ calculated according to the maximum error criterion $\left(10 \% ; t^{*}\right.$ ranging 12.5-30 min depending on the brain region). The total volume of distribution based on image-derived input function $\left(V_{\mathrm{T}}\right.$ (IDIF) was calculated as a non-invasive surrogate of the $V_{\mathrm{T}}$ given the high linear correlation when compared to the invasive approach $\left(r=0.977, r^{2}=0.954, p<0.0001\right)$ as we recently validated [19].

The IDIF was obtained as recently described [19]. Briefly, using the CT image for anatomical information, a sphere ( $3.5 \mathrm{~mm}$ in diameter) was generated with centre in the lumen of the left ventricle of the heart. Next, by selecting the early PET frame exhibiting maximal activity, a threshold set to $50 \%$ of max was applied in order to obtain a stable volume across subjects and standardize the procedure. The volume was then used to extract the activity directly from the PET image. The IDIF measured for each genotype and time point are shown in Supplementary Fig. 1. No radiometabolite correction was applied to the IDIF as no difference in metabolism between genotypes was measured (Supplementary Fig. 2).

Also, since an excellent agreement exists between values obtained with 2TCM and Logan plot $\left(r=0.993, r^{2}=0.986\right.$, $p<0.0001$ ) [19], only $V_{\mathrm{T}}$ (IDIF) values determined with Logan are reported. Finally, to investigate the consequence of applying a reference region-based analysis on mGluR1 quantification, we estimated the binding potential $\left(B P_{\mathrm{ND}}\right)$ using pons as reference region as it was previously reported [29]. In addition to the distribution volume ratio minus 1 (DVR-1) [30], calculated as the ratio of $V_{\mathrm{T}}$ (IDIF) of a target region over the $V_{\mathrm{T}}$ (IDIF) in the reference region minus 1 , we used the simplified reference tissue model 2 (SRTM2) [31]. Given the agreement between the two approaches $(\mathrm{r}=0.992$, $\left.\mathrm{r}^{2}=0.984, p<0.0001\right)$, only $B P_{\mathrm{ND}}$ values based on SRTM2 are reported. The simplified reference tissue model (SRTM) [32] was not included as it failed to fit the data of few subjects. For this reason, the $k_{2}$ ' values for SRTM2 were based on the average value of each group across different regions.

Parametric $V_{\mathrm{T}}$ (IDIF) maps were generated in PXMOD (Pmod Technologies, Zurich, Switzerland) through voxelwise Logan graphical analysis using the IDIF as input function with the $t^{*}$ calculated according to the maximum error criterion $(10 \%)$. The averages for each genotype were overlaid to a 3D mouse brain template for anatomical reference. Finally, PET images were smoothed using an isotropic Gaussian filter $(\mathrm{FWHM}=0.5 \mathrm{~mm})$, and a voxel-wise analysis with statistical parametric mapping (SPM) was performed using SPM12 (Wellcome Department of Imaging Neuroscience, London, UK). For voxel-wise analysis, data obtained from WT and HET Q175DN mice at each time point were compared, considering both contrasts (WT $>$ HET and HET $>$ WT). Statistical T-maps were calculated for a peak voxel threshold of $p=0.05$ (uncorrected) and cluster threshold of 100 voxels $\left(0.8 \mathrm{~mm}^{3}\right)$. Only significant clusters with $p<0.05$ were considered and reported. 


\section{Brain Tissue Collection}

One week following the last PET scan, 16-month old animals (WT: $n=20$; HET: $n=18$ ) were euthanized by decapitation while under anaesthesia, and brains were snap-frozen in 2metylbuthane at $-35{ }^{\circ} \mathrm{C}$ for $2 \mathrm{~min}$ and further preserved at $-80^{\circ} \mathrm{C}$ until use. Sagittal sections (20 $\mu \mathrm{m}$ of thickness) were collected serially starting at $0.96 \mathrm{~mm}$ lateral according to Paxinos and Franklin [33] in triplicate on Superfrost Plus slides (Thermo Fisher Scientific, USA), using a cryostat (Leica, Germany).

\section{In Vitro [ $\left.{ }^{3} \mathrm{H}\right]$ ITDM Autoradiography}

Autoradiography was performed as recently validated [19]. Briefly, sections were pre-incubated for 20 min with binding buffer (50 mM Tris-HCl buffer, $\mathrm{pH}$ 7.4, containing $2 \mathrm{mM}$ $\mathrm{CaCl}_{2}$ and $1.2 \mathrm{mM} \mathrm{MgCl}$ ). Next, $1 \mathrm{~h}$ incubation with total binding solution (TB, $0.5 \mathrm{nM}$ of $\left.\left[{ }^{3} \mathrm{H}\right] \mathrm{ITDM}\right)$ or nonspecificbinding solution $\left(\mathrm{NB}, 0.5 \mathrm{nM}\right.$ of $\left[{ }^{3} \mathrm{H}\right] \mathrm{ITDM}+1 \mu \mathrm{M}$ of cold ITDM) at room temperature. Sections were washed in $50 \mathrm{mM}$ Tris-HCl buffer on ice, followed by distilled water, and dried for $2 \mathrm{~h}$ at room temperature. Sections were exposed on imaging plates (BAS-TR2025, Fujifilm, Japan) for $90 \mathrm{~h}$ and imaged with a phosphor imager (Fuji FLA-700 image reader). The measured grey intensity was transformed into radioactivity based on intensity values obtained using tritium standards (American Radiolabeled Chemicals Inc., USA). Given the known decay-corrected molar activity of $\left[{ }^{3} \mathrm{H}\right] \mathrm{ITDM}$ and tritium standards, the calculated radioactivity was transformed into picomoles.

Regional quantification was performed using ImageJ software (National Institute of Health, USA). Binding was measured in the same regions of interest manually drawn on each section as measured on the PET images. Regional specific binding was measured in triplicate ( 3 slices) for each region. The average of each animal was used for statistical analysis.

\section{mGluR1 Immunohistochemistry}

In vitro mGluR1 levels were also visualized by immunohistochemistry. First, sections were air dried for $5 \mathrm{~min}$ and incubated with $4 \%$ paraformaldehyde (PFA) for $15 \mathrm{~min}$ as tissue postfixation. Following rinsing with phosphate-buffered saline (PBS, pH 7.4), nonspecific binding sites were blocked using 5\% normal goat serum (NGS) and $0.3 \%$ Triton X-100 in PBS for $1 \mathrm{~h}$. Next, sections were incubated with the monoclonal primary antibody anti-mGluR1 (rabbit; 1:200;\#12551, Cell Signalling Technologies) in antibody diluent containing $1 \%$ bovine serum albumin (BSA) and $0.3 \%$ Triton X-100 in PBS overnight at room temperature. The next day, sections were rinsed with PBS prior to a $1 \mathrm{~h}$ incubation with the secondary antibody (goat anti-rabbit conjugated with horseradish peroxidase; 1:1000; Jackson ImmunoResearch) in PBS. Following three washes with PBS, sections were exposed to the colorimetric diaminobenzidine reaction (DAB reagent, Dako) for $10 \mathrm{~min}$ and stopped with distilled water for $1 \mathrm{~min}$. Finally, sections were dehydrated and mounted with DPX mounting medium (Sigma).

Images covering the entire slices were acquired at $10 \times$ magnification for quantification with a high-throughput microscope (Nikon, Japan) with NIS elements software. Quantification of the mGluR1 immunoreactivity was performed using ImageJ software (National Institute of Health, USA). Images were converted into 8-bit grayscale, and an intensity threshold was applied to all images (threshold of 170 of 255). Regions-of-interest (ROIs) were manually drawn on each image, and the percentage of positive area after thresholding was assessed. Quantification was done in triplicate (3 slices) for each region, and the average was used for statistical analysis.

\section{Statistical Analysis}

The Shapiro-Wilk test confirmed the normal distribution of the data. Longitudinal analysis of the PET data was performed using a linear mixed-model, given its robustness and the possibility to include subjects with missing observations. The linear mixed-model analysis was performed using $V_{\mathrm{T}}$ (IDIF) values as the dependent variable for each region separately, with genotype (WT and HET), time (6, 12, and 16 months), and the interaction between genotype and time (genotype*time) as fixed effects, and with subjects included as random effect, followed by multiple comparison correction for differences within and between genotypes performed using the Tukey-Kramer test. Regular two-way ANOVA with post hoc Bonferroni correction for multiple comparisons was applied to investigate differences between genotypes for postmortem variables and reference region-based quantification. Linear mixed-model analysis was performed in JMP Pro 13 (SAS), while all the other analyses were performed with GraphPad Prism (v 6.0) statistical software. Data are represented as mean \pm standard deviation (SD) unless specified otherwise; all tests were two-tailed, except for the voxel-based analyses. Statistical significance was set at $p<0.05$.

\section{Results}

\section{In Vivo mGluR1 Availability Was Higher in HD Mice}

Mean parametric $V_{\mathrm{T}}$ (IDIF) Logan maps for WT and HET mice at each investigated time point are shown in Fig. 1. HET mice were characterized by elevated brain $\left[{ }^{11} \mathrm{C}\right]$ ITDM $V_{\mathrm{T}}$ (IDIF) values compared to WT littermates (Fig. 2). At 6 months of age, HET mice displayed higher $\left[{ }^{11} \mathrm{C}\right]$ ITDM 
Fig. 1 Average $V_{\mathrm{T} \text { (IDIF) }}$ (Logan) parametric maps of $\left[{ }^{11} \mathrm{C}\right]$ ITDM in WT and HET mice at each time point. Parametric maps are overlaid onto MRI mouse brain template for anatomical localization. WT: $n=19-21$; HET: $n=18-19$. $\mathrm{WT}=$ wild-type, $\mathrm{HET}=$ heterozygous, $\mathrm{M}=$ months

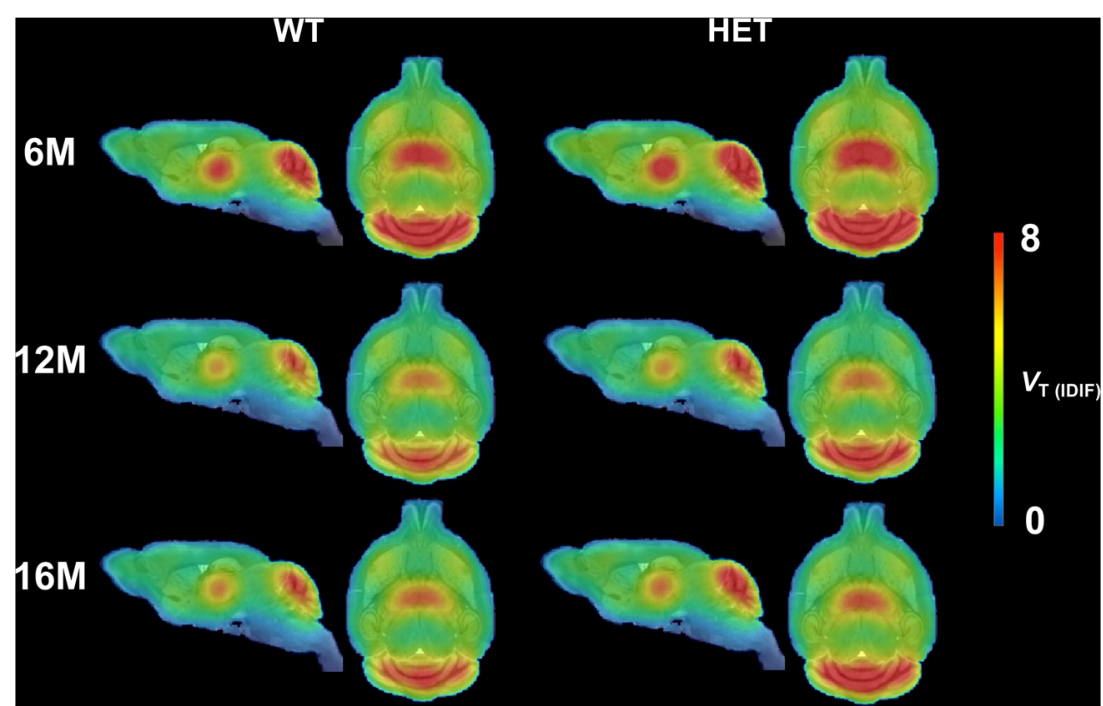

$V_{\mathrm{T} \text { (IDIF) }}$ values than WT littermates in all investigated brain regions (e.g. cerebellum: $\mathrm{WT}=6.61 \pm 0.74 \mathrm{~mL} / \mathrm{cm}^{3}$; HET $\left.=7.61 \pm 1.32 \mathrm{~mL} / \mathrm{cm}^{3} ;+15.1 \%, p<0.0001\right)$, with the exception of striatum $\left(\mathrm{WT}=4.49 \pm 0.43 \mathrm{~mL} / \mathrm{cm}^{3}\right.$; HET $=$ $\left.4.69 \pm 0.63 \mathrm{~mL} / \mathrm{cm}^{3} ;+4.4 \%, p>0.05\right)$. At 12 months of age, the $\left[{ }^{11} \mathrm{C}\right]$ ITDM $V_{\mathrm{T}}$ (IDIF) values in HET mice were elevated compared to WT littermates only in cerebellum, thalamus, and pons: e.g., cerebellar $V_{\mathrm{T}}$ (IDIF) values were $5.48 \pm$ $0.93 \mathrm{~mL} / \mathrm{cm}^{3}$ for WT mice and $6.61 \pm 0.74 \mathrm{~mL} / \mathrm{cm}^{3}$ for HET littermates $(+17.9 \%, p<0.001)$. Finally, $\left[{ }^{11} \mathrm{C}\right] \mathrm{ITDM}$ PET imaging at 16 months of age revealed that $V_{\mathrm{T}}$ (IDIF) values for HET mice were higher in cerebellum, motor cortex, hippocampus, and pons compared to WT littermates (e.g. cerebellum: $V_{\mathrm{T}}$ (IDIF) values of $5.66 \pm 0.72 \mathrm{~mL} / \mathrm{cm}^{3}$ for $\mathrm{WT}$ mice and $6.66 \pm 0.65 \mathrm{~mL} / \mathrm{cm}^{3}$ for HET littermates (+ $17.6 \%, p<0.0001$ ) (Fig. 2).

Fig. 2 Regional $\left[{ }^{11} \mathrm{C}\right] \mathrm{ITDM} V_{\mathrm{T}}$ (IDIF) quantification. HET mice demonstrated higher $\left[{ }^{11} \mathrm{C}\right] \mathrm{ITDM}$ $V_{\mathrm{T} \text { (IDIF) }}$ values compared to WT littermates. Both genotypes displayed an age-related temporal decline. Black stars indicate significance between genotypes, red stars within HET, and blue stars within WT. WT: $n=19-21$; HET: $n=18-19$. $* p<0.05, * * p<0.01$, $* * * p<0.001, * * * * p<0.0001$. WT $=$ wild-type, HET $=$ heterozygous, $\mathrm{M}=$ months
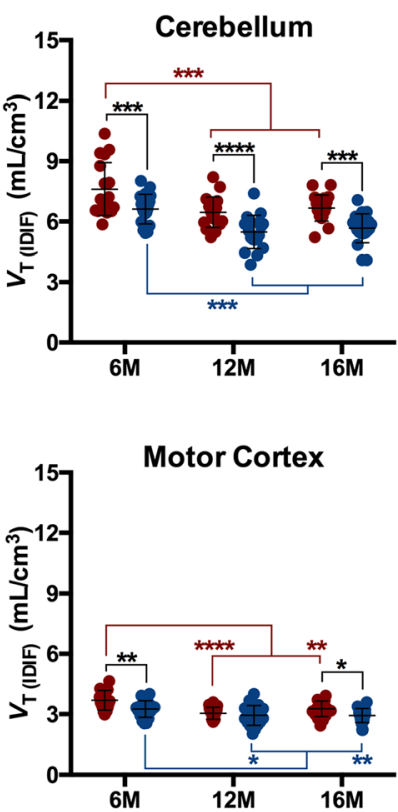

A significant temporal decline in $\left[{ }^{11} \mathrm{C}\right] \mathrm{ITDM} V_{\mathrm{T}}$ (IDIF) values was observed at 12 months of age in all investigated brain regions independently of the genotype. Accordingly, cerebellar $V_{\mathrm{T}}$ (IDIF) values between 6 and 12 months of age were reduced in both WT $(-17.2 \%, p<0.001)$ and HET $(-$ $15.1 \%, p<0.001)$ mice. No further change was observed between 12 and 16 months of age (Fig. 2).

Voxel-wise analysis of the parametric maps confirmed the extensive higher $\left[{ }^{11} \mathrm{C}\right] \mathrm{ITDM}$ binding in HET mice. For instance, in line with the regional analysis, motor cortex displayed pronounced clusters of greater $\left[{ }^{11} \mathrm{C}\right]$ ITDM binding in HET mice at 6 months of age, which were not present at 12 months, but could be detected again at 16 months of age (Fig. 3). Intriguingly, voxel-wise analysis detected clusters of lower $\left[{ }^{11} \mathrm{C}\right]$ ITDM binding in striatum of HET mice at 12 and
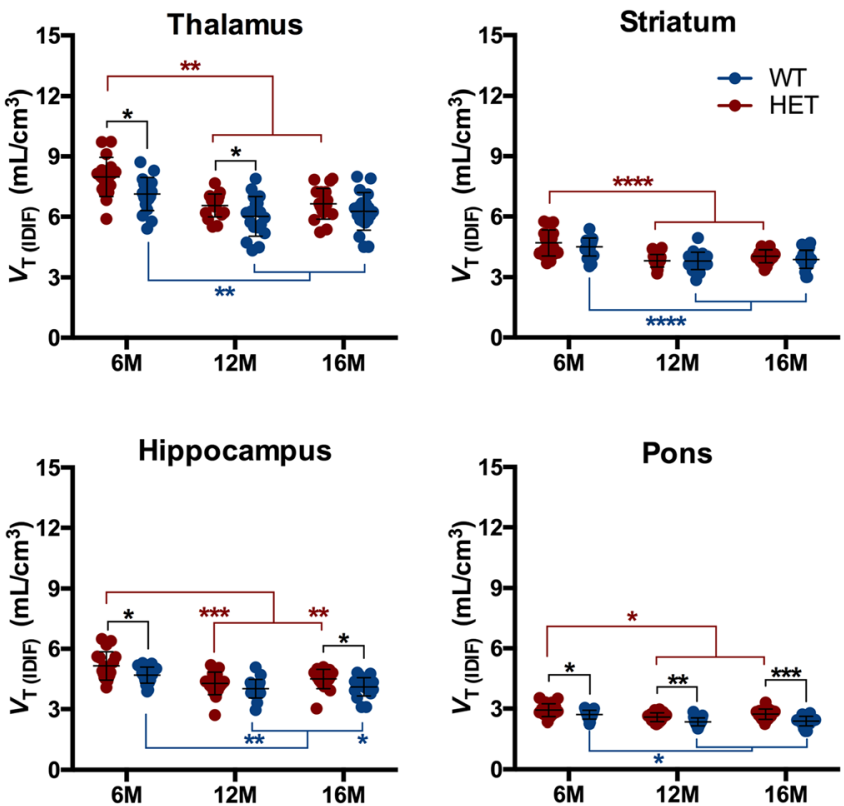


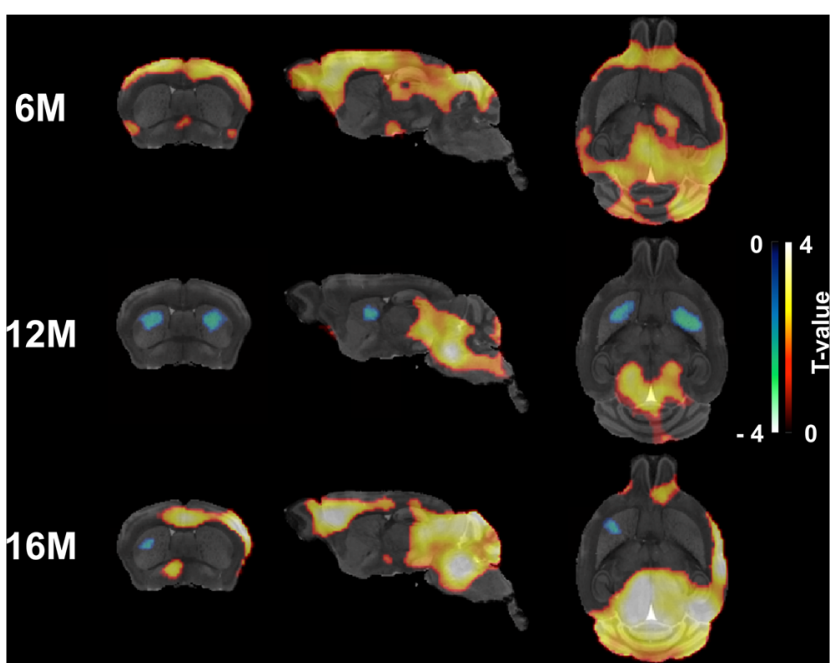

Fig. 3 Voxel-wise differences in [ ${ }^{11} \mathrm{C}$ ITDM binding between WT and HET mice. Clusters of increased $\left[{ }^{11} \mathrm{C}\right] \mathrm{ITDM}$ uptake in HET mice compared to WT littermates (HET $>$ WT) are shown as hot scale, while clusters of reduced uptake in HET mice (WT > HET) as cold scale. WT: $n=19-21$; HET: $n=18-19$. $\mathrm{M}=$ months

16 months of age (Fig. 3), which could not be detected with the regional analysis.

\section{In Vitro mGluR1 Levels Were Greater in HD Mice}

Post-mortem quantification of mGluR1 was achieved using both $\left[{ }^{3} \mathrm{H}\right] \mathrm{ITDM}$ autoradiography and mGluR1 immunohistochemistry in the same animals (WT, $n=19$; HET, $n=18$ ). Representative $\left[{ }^{3} \mathrm{H}\right] \mathrm{ITDM}$ autoradiograms and mGluR1 immunoreactivity for WT and HET mice are shown in Fig. 4a and $b$, respectively. $\left[{ }^{3} \mathrm{H}\right] \mathrm{ITDM}$-specific binding was greater in cerebellum, striatum, motor cortex, and hippocampus of HET mice compared to WT littermates (Fig. 4c) (e.g. in cerebellum:
$0.24 \pm 0.04 \mathrm{pmol} / \mathrm{mg}$ for WT; $0.29 \pm 0.04 \mathrm{pmol} / \mathrm{mg}$ for HET; $+21.7 \%, p=0.010$ ).

mGluR1 immunoreactivity, measured as percentage of positive area after thresholding, was significantly higher in cerebellum, thalamus, striatum, and motor cortex of HET mice compared to WT littermates (Fig. 4d) (e.g. in cerebellum: $49.9 \pm 5.8 \%$ for WT; $57.2 \pm 3.3 \%$ for HET; $+14.6 \%, p=$ 0.021 ).

\section{Reference Region-Based Analysis Biased the In Vivo mGluR1 Quantification}

In order to determine the effect of reference region-based quantification, we applied this approach to the time point in which we measured the largest genotypic difference (16 months of age). As shown in Fig. 5, relative quantification using pons as reference region resulted in contradictory results with HET mice displaying significantly lower values in thalamus $(-13.2 \%, p<0.001)$ and striatum $(-24.9 \%, p<0.01)$ compared to WT littermates.

\section{Discussion}

To our knowledge, this is the first study to characterize temporal mGluR1 availability in HD. We used PET imaging with the radioligand $\left[{ }^{11} \mathrm{C}\right] \mathrm{ITDM}$ to investigate changes in mGluR1 availability in HD mice during disease progression. Longitudinal PET imaging demonstrated an overall higher cerebral $\left[{ }^{11} \mathrm{C}\right] \mathrm{ITDM}$ binding in HET mice compared to WT littermates at different stages of disease progression with the exception of striatum. The most pronounced difference was found in cerebellum, with higher mGluR1 levels ranging from $15.1 \%$ to $17.9 \%$ during HD progression. Of note, only a
Fig. 4 Post-mortem evaluation of mGluR1 at 16 months of age. Representative a $\left[{ }^{3} \mathrm{H}\right] \mathrm{ITDM}$ autoradiograms and $\mathbf{b}$ mGluR1 immunohistochemistry for WT and HET mice. $\mathbf{c}\left[{ }^{3} \mathrm{H}\right] \mathrm{ITDM}$ specific binding as well as $\mathbf{d}$ mGluR1 immunoreactivity were higher in HET mice compared to WT littermates. WT: $n=17-19$; HET: $n=17-18 . * p<0.05$, $* * p<0.01, * * * p<0.001$, $* * * * p<0.0001$. Scale bar $=$ $2 \mathrm{~mm}$. WT = wild-type, $\mathrm{HET}=$ heterozygous, $\mathrm{CB}=$ cerebellum, THAL $=$ thalamus, $\mathrm{STR}=$ striatum, $\mathrm{MC}=$ motor cortex, $\mathrm{HC}=$ hippocampus

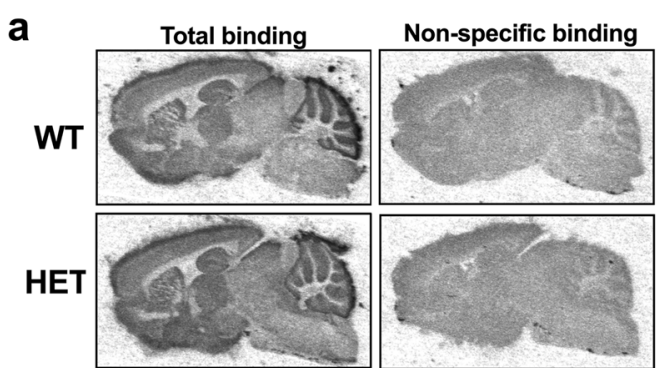

b
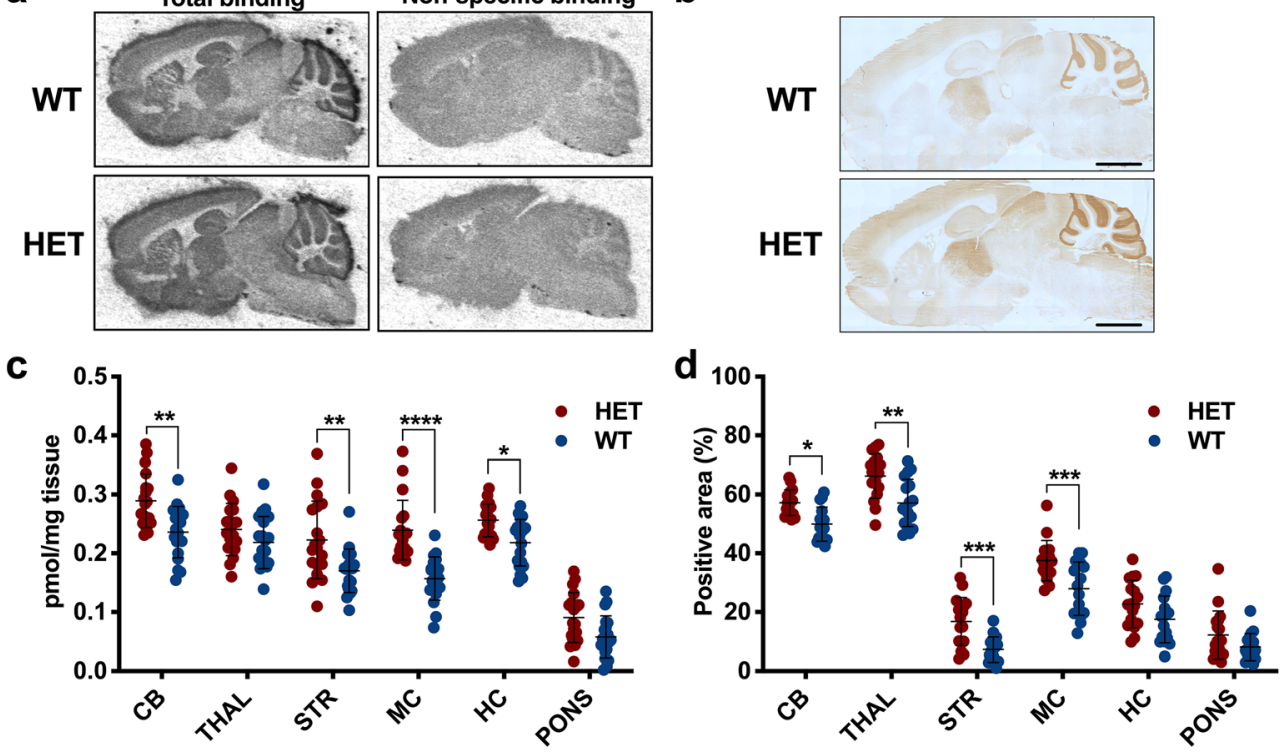


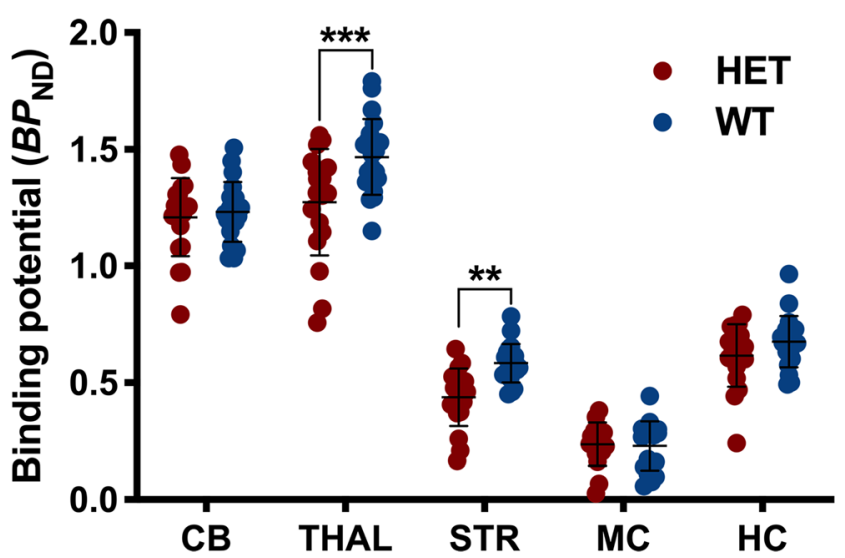

Fig. 5 Effect of reference region-based analysis on the in vivo $\left[{ }^{11}\right.$ C]ITDM quantification. Relative quantification using pons as reference region resulted in contradictory results with higher binding potential in WT mice compared to HET littermates. Data at 16 months of age are reported. $* * p<0.01, * * * p<0.001$. WT $=$ wild-type, HET $=$ heterozygous, $\mathrm{CB}=$ cerebellum, $\mathrm{THAL}=$ thalamus, $\mathrm{STR}=$ striatum, $\mathrm{MC}=$ motor cortex, $\mathrm{HC}=$ hippocampus

genotype-independent temporal decline in $\left[{ }^{11} \mathrm{C}\right] \mathrm{ITDM} V_{\mathrm{T}}$ (IDIF) was observed, indicating no disease-related effect. The observed decline is in agreement with previous investigation of the mGluR1 protein levels in the mouse cerebellum, in which a significant decline associated with age was demonstrated [34].

Only one report has previously examined in vivo mGluR1 availability in a mouse model of HD [29]. In contrast to our study, the authors reported a significant decrease in $\left[{ }^{11} \mathrm{C}\right] \mathrm{ITDM}$ binding in the R6/2 model of HD; however, these results were obtained using a reference region-based (pons) kinetic modelling for quantification of tracer binding. Our group recently found that the pons is not a suitable reference region for $\left[{ }^{11} \mathrm{C}\right]$ ITDM as its binding in this region can be blocked and displaced both in vivo and in vitro [19]. Thus, application of reference tissue models can lead to incorrect results.

Post-mortem analysis showed a significant increase in $\left[{ }^{3} \mathrm{H}\right] \mathrm{ITDM}$-specific binding as well as greater mGluR1 immunoreactivity. This consistently supports the increase in mGluR1 levels of our in vivo $\left[{ }^{11} \mathrm{C}\right]$ ITDM PET imagingbased study. No post-mortem clinical investigation of mGluR1 has been performed yet, and only sparse evidence exists on the role of mGluR1 in HD at the preclinical level. Previous autoradiography studies investigating changes in the level of group I mGluRs were inconclusive, reporting only small trends, in both the R6/2 [35] and YAC128 [36] transgenic models of HD. Interestingly, we previously showed mGluR5 protein levels to be decreased in Q175 HD mice [17], while here, we demonstrated an increase in mGluR1 levels. Because of the opposing direction of mGluR1 and mGluR5 expression levels, previous studies on Group I mGluRs may have failed to detect differences due to the lack of subtype specificity.
One potential limitation of the current study was the lack of post-mortem validation at 6 and 12 months of age using satellite cohorts of animals. While such analysis could have provided a further indication of the temporal decline in mGluR1 levels measured in vivo, it was not performed as the aim of the post-mortem work was to confirm in vitro the changes measured in vivo in the same animals.

Interestingly, the voxel-wise PET analysis detected a selective decrease of in vivo striatal mGluR1 availability in HET mice primarily at 12 months with a small cluster still present at 16 months. In humans, mHTT accumulation originates in the striatum and progresses to other brain regions [37]. Since striatal medium spiny neurons are particularly vulnerable to mHTT-induced increased levels of intracellular $\mathrm{Ca}^{2+}$, the process of receptor desensitization and internalization [38] may be enhanced in order to compensate for the several glutamatergic inputs from the basal ganglia, thalamus, and cortex [39]. This would represent a protective process as previously postulated [10] and could explain the focal cluster of decreased in vivo mGluR1 availability observed in striatum with the voxel-based analysis. Conversely, post-mortem analyses showed increased striatal mGluR1 levels. This in vivo and in vitro restricted discrepancy may be a further indication that striatal mGluR1 is undergoing internalization in order to reduce the intracellular toxic levels of $\mathrm{Ca}^{2+}$.

A complex interplay exists between HTT and mGluR1/ 5. HTT and HTT-associated protein 1 have been shown to regulate the levels of intracellular $\mathrm{Ca}^{2+}$ by interacting with $\mathrm{IP}_{3} \mathrm{R} 1$ [40]. On one hand, mHTT enhances this signalling pathway, resulting in toxic levels of intracellular $\mathrm{Ca}^{2+}$ and intensified activation of protein kinase $\mathrm{C}$ (PKC), which desensitize the mGluR $1 / 5$ receptors $[40,41]$. On the other hand, PKC also increases other downstream pathways including extracellular regulated kinase (ERK) and protein kinase B (also known as Akt) [42], which may promote cell survival and facilitate clearance of mHTT species. In addition, mHTT impairs the functionality of the Rab8/ optineurin complex [43]. Since Rab8 modulates the trafficking and signalling of mGluR1 [44], the observed mGluR1 increase may represent a combination of an adaptive response to promote cell survival and impairment in regulation of mGluR1 surface localization. In this perspective, mGluR1 has the potential to represent a therapeutic target analogously to mGluR 5 as recently reported $[12,45$, 46]. Nonetheless, due to the intricate mGluR $1 / 5$ downstream signalling pathways, it is challenging to interpret the pathological effect of these changes. Further research is needed to gain a better understanding of the potential therapeutic significance of the measured mGluR1 changes during the course of disease in this HD model and to validate these findings clinically. 


\section{Conclusion}

We report quantification of in vivo mGluR 1 availability using $\left[{ }^{11} \mathrm{C}\right]$ ITDM PET imaging during disease course in the Q175DN mouse model of HD. These findings demonstrated higher $\left[{ }^{11} \mathrm{C}\right]$ ITDM binding in extra-striatal brain regions during disease progression in HD mice, further corroborating HD as a complex whole brain disorder.

Acknowledgements The authors thank Philippe Joye, Caroline Berghmans, Eleni Van der Hallen, and Annemie Van Eetveldt of the Molecular Imaging Center Antwerp (MICA) for their valuable technical support.

Authors' Contribution DB, JV, LW, SStr, LM, CD, MS, LL, IMS, and SSta designed the study. ŠK, KC, and LW synthesized the radioligand. $\mathrm{DB}, \mathrm{JV}$, and $\mathrm{AM}$ acquired and processed the data. $\mathrm{DB}$ and JV analysed the data. DB, JV, LW, LM, CD, MS, LL, IMS, and SSta contributed to the interpretation of the data. DB, JV, and SStr wrote the manuscript. All authors assisted in reviewing the manuscript and approved its final form.

Funding Information $\mathrm{DB}$ has a PhD fellowship from the Research Foundation Flanders (FWO, 11W2516N/11W2518N). Antwerp University, Belgium founded the work through a partial assistant professor position for JV and LW, and a full professor position for SStr and SSta. LW and SStr are also supported by Antwerp University Hospital, Belgium through a departmental position.

\section{Compliance with Ethical Standards}

Conflict of Interest The Antwerp University group received financial support from CHDI Foundation, Inc., a non-profit biomedical research organization exclusively dedicated to collaboratively developing therapeutics that will substantially improve the lives of HD-affected individuals. LM, CD, LL, MS, IMS are employees of CHDI foundation, Inc. The authors declare no other potential conflicts of interest relevant to this work.

Ethical Approval All applicable institutional and/or national guidelines for the care and use of animals were followed. Experiments were performed according to the European Committee Guidelines (decree 2010/63/CEE) and the Animal Welfare Act (7 USC 2131), and they were approved by the Ethical Committee for Animal Testing (ECD 2017-27) at the University of Antwerp (Belgium).

Open Access This article is licensed under a Creative Commons Attribution 4.0 International License, which permits use, sharing, adaptation, distribution and reproduction in any medium or format, as long as you give appropriate credit to the original author(s) and the source, provide a link to the Creative Commons licence, and indicate if changes were made. The images or other third party material in this article are included in the article's Creative Commons licence, unless indicated otherwise in a credit line to the material. If material is not included in the article's Creative Commons licence and your intended use is not permitted by statutory regulation or exceeds the permitted use, you will need to obtain permission directly from the copyright holder. To view a copy of this licence, visit http://creativecommons.org/licenses/by/4.0/.

\section{References}

1. Ferraguti F, Crepaldi L, Nicoletti F (2008) Metabotropic glutamate 1 receptor: Current concepts and perspectives. Pharmacol Rev 60(4):536-581. https://doi.org/10.1124/pr.108.000166

2. Francesconi A, Duvoisin RM (1998) Role of the second and third intracellular loops of metabotropic glutamate receptors in mediating dual signal transduction activation. J Biol Chem 273(10):56155624

3. Ribeiro FM, Paquet M, Cregan SP, Ferguson SS (2010) Group I metabotropic glutamate receptor signalling and its implication in neurological disease. CNS Neurol Disord Drug Targets 9(5):574 595

4. Ribeiro FM, Pires RG, Ferguson SS (2011) Huntington's disease and group I metabotropic glutamate receptors. Mol Neurobiol 43(1):1-11. https://doi.org/10.1007/s12035-010-8153-1

5. Group THsDCR (1993) A novel gene containing a trinucleotide repeat that is expanded and unstable on Huntington's disease chromosomes. The Huntington's disease collaborative research group. Cell 72(6):971-983

6. Ross CA, Tabrizi SJ (2011) Huntington's disease: From molecular pathogenesis to clinical treatment. Lancet Neurol 10(1):83-98. https://doi.org/10.1016/S1474-4422(10)70245-3

7. Ross CA, Aylward EH, Wild EJ, Langbehn DR, Long JD, Warner JH, Scahill RI, Leavitt BR et al (2014) Huntington disease: Natural history, biomarkers and prospects for therapeutics. Nat Rev Neurol 10(4):204-216. https://doi.org/10.1038/nrneurol.2014.24

8. Abe T, Sugihara H, Nawa H, Shigemoto R, Mizuno N, Nakanishi S (1992) Molecular characterization of a novel metabotropic glutamate receptor mGluR5 coupled to inositol phosphate/Ca2+ signal transduction. J Biol Chem 267(19):13361-13368

9. Fotuhi M, Sharp AH, Glatt CE, Hwang PM, von Krosigk M, Snyder SH, Dawson TM (1993) Differential localization of phosphoinositide-linked metabotropic glutamate receptor (mGluR1) and the inositol 1,4,5-trisphosphate receptor in rat brain. J Neurosci 13(5):2001-2012

10. Ribeiro FM, Paquet M, Ferreira LT, Cregan T, Swan P, Cregan SP, Ferguson SS (2010) Metabotropic glutamate receptor-mediated cell signaling pathways are altered in a mouse model of Huntington's disease. J Neurosci 30(1):316-324. https://doi.org/10.1523/ JNEUROSCI.4974-09.2010

11. Gulyas B, Sovago J, Gomez-Mancilla B, Jia Z, Szigeti C, Gulya K, Schumacher M, Maguire RP et al (2015) Decrease of mGluR5 receptor density goes parallel with changes in enkephalin and substance P immunoreactivity in Huntington's disease: A preliminary investigation in the postmortem human brain. Brain Struct Funct 220(5):3043-3051. https://doi.org/10.1007/s00429-014-0812-y

12. Abd-Elrahman KS, Hamilton A, Hutchinson SR, Liu F, Russell RC, Ferguson SSG (2017) mGluR5 antagonism increases autophagy and prevents disease progression in the zQ175 mouse model of Huntington's disease. Sci Signal 10(510). https://doi.org/10.1126/ scisignal.aan6387

13. Ribeiro FM, Devries RA, Hamilton A, Guimaraes IM, Cregan SP, Pires RG, Ferguson SS (2014) Metabotropic glutamate receptor 5 knockout promotes motor and biochemical alterations in a mouse model of Huntington's disease. Hum Mol Genet 23(8):2030-2042. https://doi.org/10.1093/hmg/ddt598

14. Doria JG, de Souza JM, Andrade JN, Rodrigues HA, Guimaraes IM, Carvalho TG, Guatimosim C, Dobransky T et al (2015) The mGluR5 positive allosteric modulator, CDPPB, ameliorates pathology and phenotypic signs of a mouse model of Huntington's disease. Neurobiol Dis 73:163-173. https://doi.org/10.1016/j.nbd. 2014.08.021

15. Doria JG, de Souza JM, Silva FR, Olmo IG, Carvalho TG, AlvesSilva J, Ferreira-Vieira TH, Santos JT et al (2018) The mGluR5 
positive allosteric modulator VU0409551 improves synaptic plasticity and memory of a mouse model of Huntington's disease. $\mathrm{J}$ Neurochem 147(2):222-239. https://doi.org/10.1111/jnc.14555

16. Menalled LB, Kudwa AE, Miller S, Fitzpatrick J, Watson-Johnson J, Keating N, Ruiz M, Mushlin R et al (2012) Comprehensive behavioral and molecular characterization of a new knock-in mouse model of Huntington's disease: zQ175. PLoS One 7(12):e49838. https://doi.org/10.1371/journal.pone.0049838

17. Bertoglio D, Kosten L, Verhaeghe J, Thomae D, Wyffels L, Stroobants S, Wityak J, Dominguez C et al (2018) Longitudinal characterization of mGluR5 using (11)C-ABP688 PET imaging in the Q175 mouse model of Huntington's disease. J Nucl Med. https://doi.org/10.2967/jnumed.118.210658

18. Fujinaga M, Yamasaki T, Maeda J, Yui J, Xie L, Nagai Y, Nengaki $\mathrm{N}$, Hatori A et al (2012) Development of N-[4-[6(isopropylamino)pyrimidin-4-yl]-1,3-thiazol-2-yl]-N-methyl-4$[11 \mathrm{C}]$ methylb enzamide for positron emission tomography imaging of metabotropic glutamate 1 receptor in monkey brain. J Med Chem 55(24):11042-11051. https://doi.org/10.1021/jm301597s

19. Bertoglio D, Verhaeghe J, Korat S, Miranda A, Wyffels L, Stroobants S, Mrzljak L, Dominguez C et al (2019) in vitro and in vivo assessment of suitable reference region and kinetic modelling for the mGluR1 radioligand [11C]ITDM in mice. Mol Imaging Biol. https://doi.org/10.1007/s11307-019-01435-1

20. Cudalbu C, McLin VA, Lei H, Duarte JM, Rougemont AL, Oldani G, Terraz S, Toso C et al (2013) The C57BL/6J mouse exhibits sporadic congenital portosystemic shunts. PLoS One 8(7):e69782. https://doi.org/10.1371/journal.pone.0069782

21. Peng Q, Wu B, Jiang M, Jin J, Hou Z, Zheng J, Zhang J, Duan W (2016) Characterization of behavioral, Neuropathological, brain metabolic and key molecular changes in zQ175 knock-in mouse model of Huntington's disease. PLoS One 11(2):e0148839. https://doi.org/10.1371/journal.pone.0148839

22. Heikkinen T, Lehtimaki K, Vartiainen N, Puolivali J, Hendricks SJ, Glaser JR, Bradaia A, Wadel K et al (2012) Characterization of neurophysiological and behavioral changes, MRI brain volumetry and $1 \mathrm{H}$ MRS in zQ175 knock-in mouse model of Huntington's disease. PLoS One 7(12):e50717. https://doi.org/10.1371/journal. pone.0050717

23. Haggkvist J, Toth M, Tari L, Varnas K, Svedberg M, Forsberg A, Nag S, Dominguez C et al (2016) Longitudinal microPET imaging of the $\mathrm{zQ} 175$ mouse model of Huntington's disease shows in vivo changes of molecular targets in the striatum and the cerebral cortex. J Nucl Med. https://doi.org/10.2967/jnumed.116.180497

24. Bertoglio D, Verhaeghe J, Kosten L, Thomae D, Van der Linden A, Stroobants S, Wityak J, Dominguez C et al (2018) MR-based spatial normalization improves [18F]MNI-659 PET regional quantification and detectability of disease effect in the Q175 mouse model of Huntington's disease. PLoS One 13(10):e0206613. https://doi. org/10.1371/journal.pone.0206613

25. Verhaeghe J, Bertoglio D, Kosten L, Thomae D, Verhoye M, Van Der Linden A, Wyffels L, Stroobants S et al (2018) Noninvasive relative quantification of [(11)C]ABP688 PET imaging in mice versus an input function measured over an Arteriovenous shunt. Front Neurol 9:516. https://doi.org/10.3389/fneur.2018.00516

26. Johnson GA, Badea A, Brandenburg J, Cofer G, Fubara B, Liu S, Nissanov J (2010) Waxholm space: an image-based reference for coordinating mouse brain research. Neuroimage 53(2):365-372. https://doi.org/10.1016/j.neuroimage.2010.06.067

27. Julien-Dolbec C, Tropres I, Montigon O, Reutenauer H, Ziegler A, Decorps M, Payen JF (2002) Regional response of cerebral blood volume to graded hypoxic hypoxia in rat brain. Br J Anaesth 89(2): 287-293

28. Logan J, Fowler JS, Volkow ND, Wolf AP, Dewey SL, Schlyer DJ, MacGregor RR, Hitzemann R et al (1990) Graphical analysis of reversible radioligand binding from time-activity measurements applied to [N-11C-methyl]-(-)-cocaine PET studies in human subjects. J Cereb Blood Flow Metab 10(5):740-747. https://doi.org/10. 1038/jcbfm.1990.127

29. Yamasaki T, Fujinaga M, Yui J, Ikoma Y, Hatori A, Xie L, Wakizaka H, Kumata K et al (2014) Noninvasive quantification of metabotropic glutamate receptor type 1 with [(1)(1)C]ITDM: A small-animal PET study. J Cereb Blood Flow Metab 34(4): 606-612. https://doi.org/10.1038/jcbfm.2013.243

30. Innis RB, Cunningham VJ, Delforge J, Fujita M, Gjedde A, Gunn RN, Holden J, Houle S et al (2007) Consensus nomenclature for in vivo imaging of reversibly binding radioligands. J Cereb Blood Flow Metab 27(9):1533-1539. https://doi.org/10.1038/sj.jcbfm. 9600493

31. Wu YJ, Carson RE (2002) Noise reduction in the simplified reference tissue model for neuroreceptor functional imaging. J Cereb Blood Flow Metab 22(12):1440-1452

32. Lammertsma AA, Hume SP (1996) Simplified reference tissue model for PET receptor studies. Neuroimage 4(3 Pt 1):153-158. https://doi.org/10.1006/nimg.1996.0066

33. Paxinos G, Franklin K (2003) The mouse brain in stereotaxic coordinates. Academic press; 2 edition (December 8, 2003),

34. Simonyi A, Xia J, Igbavboa U, Wood WG, Sun GY (1998) Age differences in the expression of metabotropic glutamate receptor 1 and inositol 1,4,5-trisphosphate receptor in mouse cerebellum. Neurosci Lett 244(1):29-32. https://doi.org/10.1016/s03043940(98)00127-x

35. Cha JH, Kosinski CM, Kerner JA, Alsdorf SA, Mangiarini L, Davies SW, Penney JB, Bates GP et al (1998) Altered brain neurotransmitter receptors in transgenic mice expressing a portion of an abnormal human Huntington disease gene. Proc Natl Acad Sci U S A 95(11):6480-6485

36. Benn CL, Slow EJ, Farrell LA, Graham R, Deng Y, Hayden MR, Cha JH (2007) Glutamate receptor abnormalities in the YAC128 transgenic mouse model of Huntington's disease. Neuroscience 147(2):354-372. https://doi.org/10.1016/j.neuroscience.2007.03. 010

37. Carty N, Berson N, Tillack K, Thiede C, Scholz D, Kottig K, Sedaghat Y, Gabrysiak C et al (2015) Characterization of HTT inclusion size, location, and timing in the zQ175 mouse model of Huntington's disease: an in vivo high-content imaging study. PLoS One 10(4):e0123527. https://doi.org/10.1371/journal.pone. 0123527

38. Ferguson SS (2001) Evolving concepts in G protein-coupled receptor endocytosis: the role in receptor desensitization and signaling. Pharmacol Rev 53(1):1-24

39. Joel D, Weiner I (1994) The organization of the basal gangliathalamocortical circuits: Open interconnected rather than closed segregated. Neuroscience 63(2):363-379

40. Tang TS, Tu H, Chan EY, Maximov A, Wang Z, Wellington CL, Hayden MR, Bezprozvanny I (2003) Huntingtin and huntingtinassociated protein 1 influence neuronal calcium signaling mediated by inositol- $(1,4,5)$ triphosphate receptor type 1 . Neuron 39(2):227239

41. Tang TS, Slow E, Lupu V, Stavrovskaya IG, Sugimori M, Llinas R, Kristal BS, Hayden MR et al (2005) Disturbed Ca2+ signaling and apoptosis of medium spiny neurons in Huntington's disease. Proc Natl Acad Sci U S A 102(7):2602-2607. https://doi.org/10.1073/ pnas.0409402102

42. Choe ES, Wang JQ (2001) Group I metabotropic glutamate receptors control phosphorylation of CREB, Elk-1 and ERK via a CaMKII-dependent pathway in rat striatum. Neurosci Lett 313(3): 129-132

43. del Toro D, Alberch J, Lazaro-Dieguez F, Martin-Ibanez R, Xifro X, Egea G, Canals JM (2009) Mutant huntingtin impairs post-Golgi trafficking to lysosomes by delocalizing optineurin/Rab8 complex 
from the Golgi apparatus. Mol Biol Cell 20(5):1478-1492. https:// doi.org/10.1091/mbc.E08-07-0726

44. Esseltine JL, Ribeiro FM, Ferguson SS (2012) Rab8 modulates metabotropic glutamate receptor subtype 1 intracellular trafficking and signaling in a protein kinase C-dependent manner. J Neurosci 32(47):16933-16942a. https://doi.org/10.1523/JNEUROSCI. 0625-12.2012

45. Abd-Elrahman KS, Ferguson SSG (2019) Modulation of mTOR and CREB pathways following mGluR5 blockade contribute to improved Huntington's pathology in zQ175 mice. Mol Brain 12(1):35. https://doi.org/10.1186/s13041-019-0456-1
46. Ribeiro FM, Hamilton A, Doria JG, Guimaraes IM, Cregan SP, Ferguson SS (2014) Metabotropic glutamate receptor 5 as a potential therapeutic target in Huntington's disease. Expert Opin Ther Targets 18(11):1293-1304. https://doi.org/10.1517/14728222. 2014.948419

Publisher's Note Springer Nature remains neutral with regard to jurisdictional claims in published maps and institutional affiliations. 